\title{
EVALUATION OF DIFFERENT PROCEDURE INVOLVED IN THE Transcranial Direct Current Stimulation (tDCS) TECHNIQUE EXPERIMENTAL APPLICATION
}

\author{
Lauren Naomi Spezia Adachi $i^{1,2,3}$, Carla de Oliveira ${ }^{1,2,3}$, Rafael Vercelino ${ }^{1,4}$, \\ Isabel Cristina de Macedo ${ }^{1,3,5}$, Gabriela Laste ${ }^{2,3}$, Alexandre Silva Quevedo ${ }^{1,6}$, \\ Vanessa Leal Scarabelot ${ }^{1,3,5}$, Wolnei Caumo ${ }^{2}$, \\ Iraci Lucena da Silva Torres , $^{1,2,5}$
}

\begin{abstract}
Introduction: The transcranial direct current stimulation (tDCS) is a non-invasive technique, which induces neuroplastic changes in the central nervous system of animals and humans. Furthermore, tDCS has been suggested as a therapeutic tool for pain management. The aim of this study was to standardize a non-invasive tDCS technique indexed by the nociceptive response of rats submitted to different conditions necessary to the tDCS application.
\end{abstract}

Method: 60 -day-old male Wistar rats $(n=65)$, divided into 6 groups: control(C); non-active sham (NAS); active-sham (AS); active-sham restrained (ASR); non-active sham restrained (NASR); active tDCS treatment. Animals received treatment during 30 seconds (sham-active) or 20 minutes (restraint and tDCS)/8 days. Nociceptive threshold was assessed by Hot Plate test at baseline, immediately and $24 \mathrm{~h}$ after the first session, immediately and $24 \mathrm{~h}$ after the last session. Variance analysis of repeated measurements followed by Bonferroni was performed for intra-group comparison.

Results: Physical restraint and 30 seconds stimulation (sham-tDCS) increased pain sensitivity $(P \leq 0.05)$, and tDCS treatment was able to prevent the thermal hyperalgesia. Our original tDCS montage is similar to that used in the procedure with humans, because it is not an invasive technique. The electrodes are positioned on the head, and the animals are immobilized during the 20-minute treatment. As this procedure could involve behavior and neurochemical alterations due to stress induced by restriction (thus, it creates a research bias), we hypothesized that a 30 -second electrical stimulus application (sham-tDCS) and the physical restriction used during tDCS treatment might alter nociceptive response in rats.

Conclusion: There are methodological limitations in the present tDCS-technique. Although active-tDCS treatment is able to prevent these harmful effects, interference of these factors has to be considered during the results' analysis. Future adaptations of the tDCS-technique in rats are required to evaluate its therapeutic effects.

Keywords: tDCS; animal model; nociception; thermal hyperalgesia; stress restraint
Clin Biomed Res. 2017;37(2):63-72

1 Laboratory of Pain Pharmacology and Neuromodulation: Preclinical Studies, Department of Pharmacology, Institute of Basic Health Sciences, Universidade Federal do Rio Grande do Sul (UFRGS) Porto Alegre, RS, Brazil.

2 Medical Sciences Graduate Program: Medical Sciences, Universidade Federal do Rio Grande Sul (UFRGS). Porto Alegre, RS, Brazil.

3 Animal Experimentation Unit and Graduate Research Group, Hospital de Clínicas de Porto Alegre, Universidade Federal do Rio Grande do Sul (UFRGS). Porto Alegre, RS, Brazil.

4 Rehabilitation Sciences Graduate Program, Universidade Federal de Ciências da Saúde de Porto Alegre (UFCSPA). Porto Alegre, RS, Brazil.

5 Biological Sciences Graduate Program: Physiology, Universidade Federal do Rio Grande do Sul (UFRGS). Porto Alegre, RS, Brazil.

6 Department of Surgery and Orthopedics, School of Dentistry, Universidade Federal do Rio Grande do Sul (UFRGS). Porto Alegre, RS, Brazil.

Corresponding author: Iraci Lucena da Silva Torres iltorres@hcpa.edu.br Department of Pharmacology, Institute of Basic Health Sciences, Universidade Federal do Rio Grande do Sul (UFRGS) Rua Sarmento Leite, 500, sala 305. 90050-170, Porto Alegre, RS, Brazil. 
Transcranial direct current stimulation (tDCS) is a non-invasive technique that has emerged as a promising treatment for several pain syndromes ${ }^{1-3}$. This technique consists in the applying of a weak, direct, constant and low intensity electric current. Interestingly, the treatment with tDCS is painless, and has been used to treat chronic pain syndromes ${ }^{3}$ and many neuropsychiatry disorders ${ }^{1,2}$. It has also been proposed that the mechanisms by which tDCS induce changes across different levels of the nervous system may involve membrane polarization and, consequently, the modulation of neuronal activity ${ }^{4}$. Besides, the effects of tDCS on cortical excitability are polarity-dependent. An interesting issue is that tDCS modifies not only the activity of cortical areas located directly under the electrodes, but also from distant areas possibly due to primary interconnections ${ }^{5}$. The current model of tDCS effects is based on cortico-cortical interactions, with some subcortical components (e.g., anterior cingulate cortex and thalamic nuclei) in those circuits ${ }^{6}$. Importantly, clinical studies have revealed that tDCS can improve cognition performance ${ }^{7}$ in stroke patients ${ }^{8}$. Likewise, tDCS is a good option to treat chronic disorders such as fibromyalgia ${ }^{9}$, chronic pain ${ }^{10}$, Parkinson's disease ${ }^{9}$, major depression ${ }^{11-13}$, and central post-stroke pain ${ }^{14}$. The changes caused by weak and constant current in the scalp alter the excitability at different levels of the nervous system ${ }^{15,16}$, probably leading to membrane potential alterations and, consequently, modulating the neuronal activity ${ }^{4}$. Nevertheless, the specificity of tDCS implies application-specific customization of protocols to maximize desired outcomes and minimize undesired effects. An important issue is that given the simplicity of tDCS and the complexity of brain function, understanding the mechanisms leading to specificity is fundamental to the advancement of $t D C S^{17}$.

Moreover, previous studies of our research group using rats have confirmed the immediate and long-lasting effects of repeated tDCS treatment on chronic pain inflammation ${ }^{18}$ and hyperalgesia induced by chronic restraint stress models ${ }^{19}$. In addition, we demonstrated that $\mathrm{DDCS}$ was able to decrease BDNF levels in the structures involved in the descending systems only in unstressed animals ${ }^{20}$. On our montage, the animals received tDCS treatment with both electrodes positioned on the head, a montage similar to a treatment for humans ${ }^{18}$. The electrodes were placed, without surgery or anesthesia; the animals were immobilized during the 20-minute treatment to prevent the electrodes removal from the head. Similar to a stress model, the immobilization of animal body could activate the sympatho-adrenal ${ }^{21}$ and pituitary-adrenocortical ${ }^{22}$ axis. Moreover, the physiological stressor may increase heart rate, blood pressure, and plasma levels of norepinephrine and epinephrine, leading to the activation of intrinsic mechanisms of pain inhibition ${ }^{23}$. Also, 30 seconds of stimulation is the parameter used for sham-tDCS in humans. Thus, we also applied this procedure in rats, mimicking the technique used in clinical trials. However, in previous studies we observed a possible sham effect on pain threshold in naive and chronic stressed animals ${ }^{20}$. Therefore, we hypothesized that forced immobilization could interfere with the tDCS effects and that the application of 30 seconds of tDCS (sham stimulation) could change the nociceptive behavior of rats.

Thus, the aim of the present study was to evaluate the influence of different procedures (restraint, active-sham tDCS - 30 seconds stimulation, and tDCS-active) that are involved in our protocol of tDCS technique in rats. This information is necessary to better adjust the application of tDCS protocol in rats for nociception conditions.

\section{METHODS}

\section{Animals}

Sixty-five male Wistar rats weighing 250-350g were used ( $n=10-15$ for group). Experimentally naive animals were housed in groups of five in home cages made of Polypropylene material $(49 \times 34 \times 16 \mathrm{~cm})$. All animals were maintained in a standard 12:12 light/dark cycle (lights on at 07:00 a.m. and lights off at 07:00 p.m.) in a temperature controlled environment $\left(22 \pm 2{ }^{\circ} \mathrm{C}\right)$. Animals had ad libitum access to water and food. All experiments and procedures were approved by the Institutional Animal Care and Use Committee (GPPG-HCPA protocol N. 10.0381) and conducted in compliance with Brazilian laws/2013 and the Laboratory Guide for the Care and Use of Animals (The National Academies Press, Eighth Edition, 2011). The husbandry of the animals followed Law No. 11794 (Brazil), which regulates the scientific use of animals. The experimental protocol complied with the ethical and methodological standards of the ARRIVE guidelines ${ }^{24}$. Vigorous attempts were made to minimize animal suffering and decrease external sources of pain and discomfort, as well as to use the minimum number of animals required to produce reliable scientific data. To control the possible effect of outliers, animals that did not present behavioral responses were excluded.

\section{Experimental Design}

Rats were habituated to the maintenance room for 1 week before the experiment started. After this period, the animals were randomly allocated in six groups: 
1 - control (C) without intervention; 2 - non-active sham (NAS): the animals were not restrained, the electrodes were positioned on the head, but the stimulator was turned off; 3 - active sham (AS): the animals were not restrained, the electrodes were positioned on the head, and the stimulator was turned on for 30 seconds; 4 - non-active sham restrained (NASR): the animals were restrained during 20 minutes, the electrodes were positioned on the head, but the stimulator was turned off; 5 - active-sham restrained (ASR): the animals were restrained during 20 minutes, the electrodes were placed on the head, and the stimulator was turned on for 30 seconds; 6 - tDCS: the animals were restrained, the electrodes were placed on the head, and the stimulator was turned on for 20 minutes (figure 1).

\section{Maintenance of Blinding}

To control for possible measurement bias in the present study, the following measures were taken: trichotomy was performed in all animals on the region where the electrodes were placed in groups treated with tDCS / Sham and control groups. In addition, there was the participation of a third researcher, who conducted the numbering of the boxes prior to the tests, without the involvement of the researcher responsible for the behavioral assessment. Therefore, the animal groups receiving active treatment with tDCS / Sham and control could not be identified. The behavioral tests were conducted by two researchers, who filmed the tests, and three researchers analyzed the results. To avoid unblinding of the outcomes evaluators, it is important to inform that these researchers were unaware of the experimental protocol. Thus, it is believed that the possibilities of influence on behavior results were eliminated.

\section{Transcranial Direct Current Stimulation (tDCS)}

The animals of the active-tDCS group received anodal tDCS therapy, which consists of a constant low intensity current $(0.5 \mathrm{~mA})$ applied for 20 minutes every afternoon during 8 days as described by Spezia Adachi et al. ${ }^{19}$ and Laste et al. ${ }^{18}$.

The direct current was delivered from a battery-driven, constant current stimulator, using silver-silver chloride $(\mathrm{Ag} / \mathrm{AgCl})$ sensor electrodes. Rat heads were shaved for better adherence of the electrodes and were trimmed to $1.5 \mathrm{~cm}^{2}$ for a better fit. The electrodes had a conductive adhesive hydrogel and were fixed onto the head using adhesive tape (Micropore ${ }^{\mathrm{TM}}$ ) and covered with a protective mesh to prevent removal. During the 20-minute session of anodal tDCS, the animals remained gently wrapped in a towel. The anodal electrode was placed between the ears, on the neck of the rat (parietal cortex), and the cathodal electrode was positioned at the midpoint between the lateral angles of both eyes (supraorbital area) $)^{18,19,25}$. This technique mimics tDCS protocols used in humans ${ }^{26,27}$, and it has been applied by our research group showing antinociceptive effects ${ }^{18,19}$.

According to an earlier study ${ }^{15}$, a constant current intensity of $1 \mathrm{~mA}$ causes skin lesions, as current density is comparatively much higher than the traditional $1 \mathrm{~mA}$ tDCS using large pads in humans. Further, a current density higher than $142.9 \mathrm{~A} / \mathrm{m} 2$ is

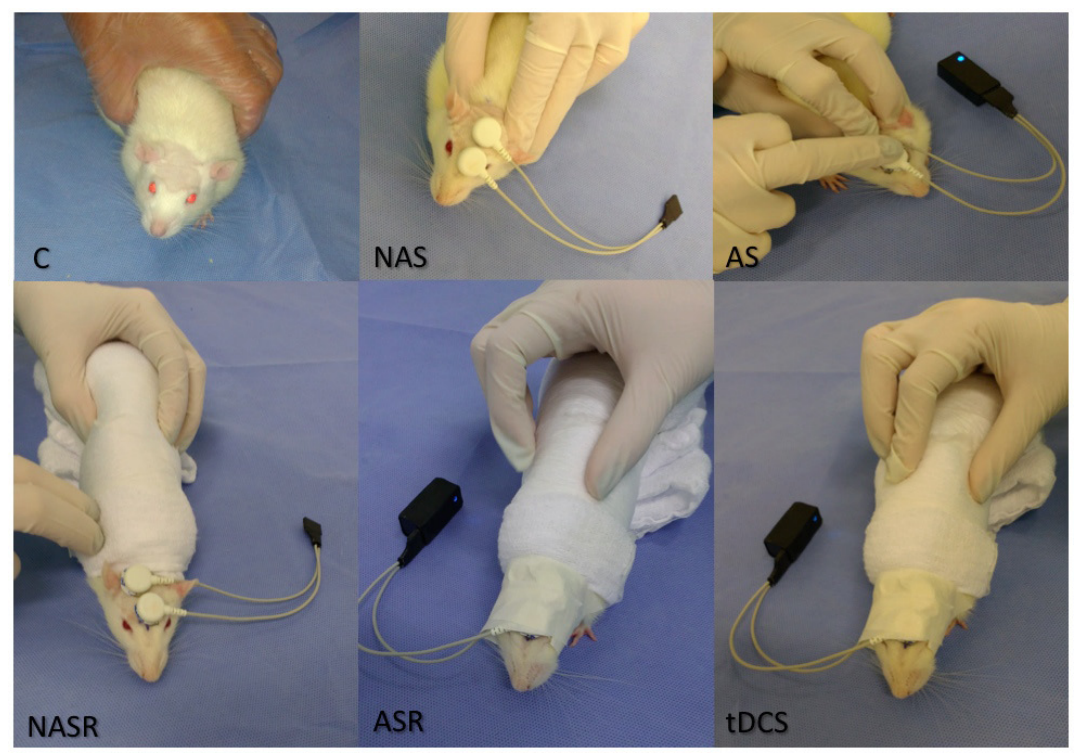

Figure 1: Experimental design of groups. C: control; NAS: non-active sham; AS: active sham; NASR: non-active sham restrained; ASR: active-sham restrained; tDCS: active tDCS treatment. 
associated with brain lesions. Based on this threshold, our stimulation parameters consisted of a current density of $33.4 \mathrm{~A} / \mathrm{m} 2^{15}$. Nevertheless, in our study, electrodes were fixed onto the skin, and we did not observe any lesions using this montage and current intensity. Notably, this model of application required no anesthesia, unlike models used in previous tDCS studies in rats ${ }^{15}$. In fact, this lack of anesthesia strengthens the study because volatile anesthesia (such as isoflurane) has been shown to decrease excitatory transmission and to increase inhibitory transmission ${ }^{28}$, altering BDNF levels and, therefore, neuroplasticity ${ }^{29}$. Thus, we removed this confounding factor by adapting the human model using $\mathrm{Ag} / \mathrm{AgCl}$ sensor electrodes ${ }^{30}$ in rats.

For the non-active sham group, the electrodes were placed in the same positions as the active tDCS stimulation; however, the stimulator was turned off and, after 30 seconds the animals returned to their respective home cages. For the active-sham group, the electrodes were placed in the same positions as the active tDCS stimulation and the stimulator was turned on just for 30 seconds so that the animals could maintain the physical sensation of active tDCS conditions $^{31}$. Then, the animals returned to their home cages. For the non-active restrained sham group, the animals were restrained for 20 minutes and the electrodes were placed in the same positions as the active tDCS stimulation, but the stimulator was turned off. For the active restrained sham group, the animals were restrained for 20 minutes, the electrodes were placed in the same positions, and stimulator was turned on for only 30 seconds.

\section{Hot Plate Test}

The hot plate test was carried out to assess the effects of tDCS on the thermal nociceptive threshold adapted from Woolfe and MacDonald ${ }^{19,32}$. The baseline measure was performed before starting the groups' protocols. This test was applied again immediately and $24 \mathrm{~h}$ after the first and last session.

The hot plate was pre-heated and kept at a temperature of $55 \pm 0.5^{\circ} \mathrm{C}$. All rats were acclimated to the hot plate for five minutes, 24 hours prior to testing, as the novelty of the apparatus itself could induce antinociception ${ }^{33}$. Rats were placed in glass funnels on the heated surface, and the nociceptive threshold was assessed by recording the time taken to first response (foot licking, jumping, or rapidly removing paws), as described by Minami et al. ${ }^{34}$. We used the hot plate test to determine changes in latency as an indicator of modifications of the supraspinal pain process ${ }^{35}$, as licking or jumping responses during this test are considered to be the result of supraspinal sensory integration ${ }^{36,37}$. The response was recorded in seconds(s) and a cutoff time of $10 \mathrm{~s}$ was used.

\section{Statistical Analyses}

Data were expressed as the mean \pm standard error of the mean (S.E.M). Variance analysis of repeated measurements (ANOVA RM) followed by Bonferroni was performed for intra-group comparison. $P$ values lower than 0.05 were reported as statistically significant. SPSS 19.0 for Windows was used for statistical analysis.

\section{RESULTS}

\section{Control Group Showed no Differences During the Experiment}

The control group (figure 2) did not present any difference in the paw withdrawal test at different times assessed $\left(F_{(4,36)}=2,29 ; P \geq 0.05\right)$.

\section{Restraint Stress Was Able to Increase Pain Sensitivity (Hyperalgesia)}

The NASR (figure 3) group demonstrated time effect $\left(F_{(4,56)}=2,83 ; P \leq 0.05\right)$, when compared to data from basal and immediately after the last session $(P \leq 0.05)$. Thus, restriction applied during 20 minutes for 8 consecutive days without active stimulation was effective in decreasing the pain threshold on the hot plate test.

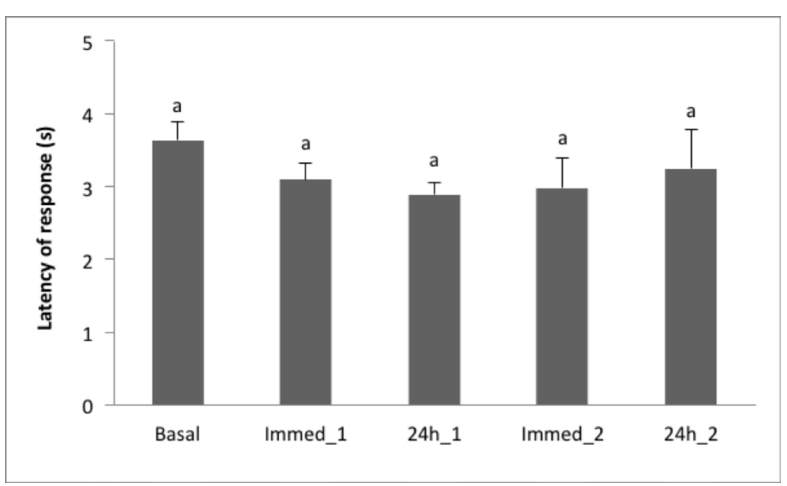

Figure 2: Evaluation of Control group at different times on hot plate test. Data were expressed as the mean \pm standard error of the mean (S.E.M). Variance analysis of repeated measurements (ANOVA RM) followed by Bonferroni was performed for intra-group comparison. The control group did not present variation at different times. Equal lowercase letters (a) indicate that there is no statistically significant difference between the different times $(P>0.05)$. Immed_1: Immediately after first session of treatment; $24 \mathrm{~h} \_1: 24 \mathrm{~h}$ after first session of treatment; Immed_2: Immediately after last session of treatment; $24 \mathrm{~h} \_2: 24 \mathrm{~h}$ after last session of treatment. 


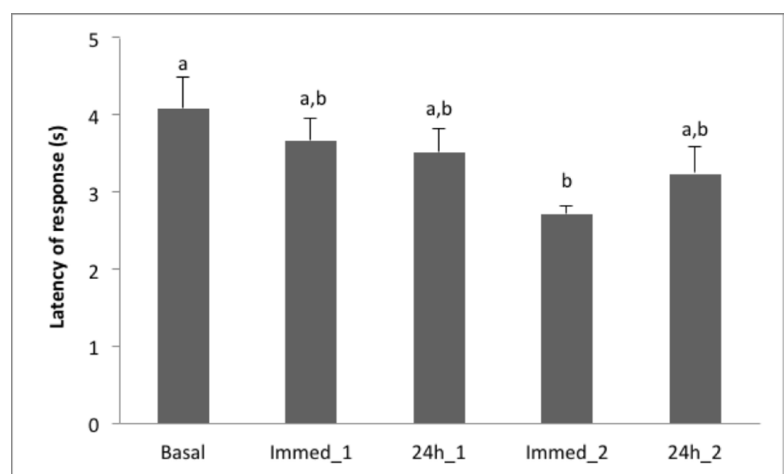

Figure 3: Evaluation of Non-active Sham Restraint group at different times on hot plate test. Data were expressed as the mean \pm standard error of the mean (S.E.M). Variance analysis of repeated measurements (ANOVA RM) followed by Bonferroni was performed for intra-group comparison. Different lowercase letters ( $a$ through $b$ ) indicate statistically significant difference between the different times $(P<0.05)$. Immed_1: Immediately after first session of treatment; 24h_1: $24 \mathrm{~h}$ after first session of treatment; Immed_2: Immediately after last session of treatment; $24 \mathrm{~h} \_2$ : $24 \mathrm{~h}$ after last session of treatment.

\section{The 30-second Electrical Stimulation (sham- tDCS) Was Able to Increase Pain Sensitivity}

The ASR group (figure 4) (which was restricted and had 30 seconds of active stimulation) demonstrated time effect $\left(F_{(4,36)}=4,92 ; P \leq 0.05\right)$ and there was a significant difference between baseline and immediately after the last session data $(P \leq 0.05)$. Likewise, the group that received 30 seconds of active stimulation and was not restricted (AS) (figure 5) also presented time effect $\left(F_{(4,36)}=6,84 ; P \leq 0.05\right)$, showing a decrease on pain threshold on the hot plate test immediately after the first $(P \leq 0.05)$ and the last $(P \leq 0.05)$ session of tDCS.

\section{The Handling of Animals Did Not Alter Pain Sensitivity}

The group of animals that was manipulated during 30 seconds without sham stimulation (NAS) (figure 6) did not show variation on pain threshold at different times of measures $\left(F_{(4,36)}=0.54 ; P \geq 0.05\right)$. These data indicate that the handling of animals during the sham-tDCS did not alter pain sensitivity.

\section{tDCS Prevented Pain Sensitivity Induced by Physical Immobilization}

The tDCS group (figure 7) did not show a variation on pain threshold at different times of measures $\left(F_{(4,36)}=3,40 ; P \geq 0.05\right)$ demonstrating that, on this montage, the tDCS was able to prevent the effect of immobilization and the effect of the first 30 seconds of stimulation.

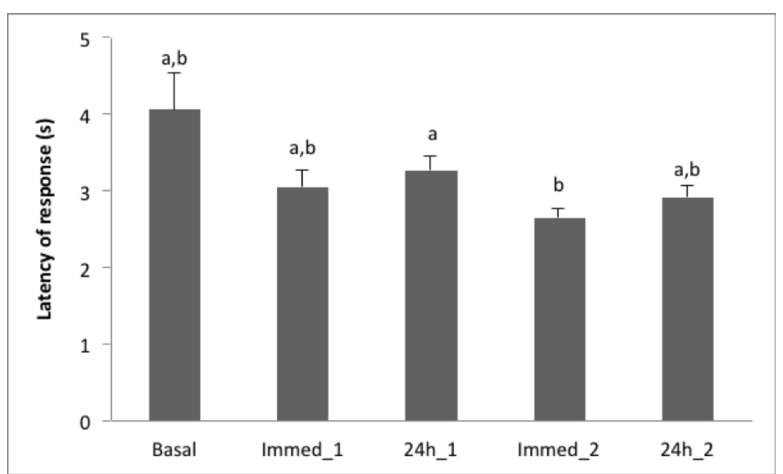

Figure 4: Evaluation of Active Sham Restraint group at different times on hot plate test. Data were expressed as the mean \pm standard error of the mean (S.E.M). Variance analysis of repeated measurements (ANOVARM) followed by Bonferroni was performed for intra-group comparison. Different lowercase letters ( $a$ through $b$ ) indicate a statistically significant difference between the different times $(\mathrm{P}<0.05)$. Immed_1: Immediately after first session of treatment; $24 \mathrm{~h} \_1: 2 \overline{\mathrm{h}}$ after first session of treatment; Immed_2: Immediately after last session of treatment; 24h_2: $24 \mathrm{~h}$ after last session of treatment.

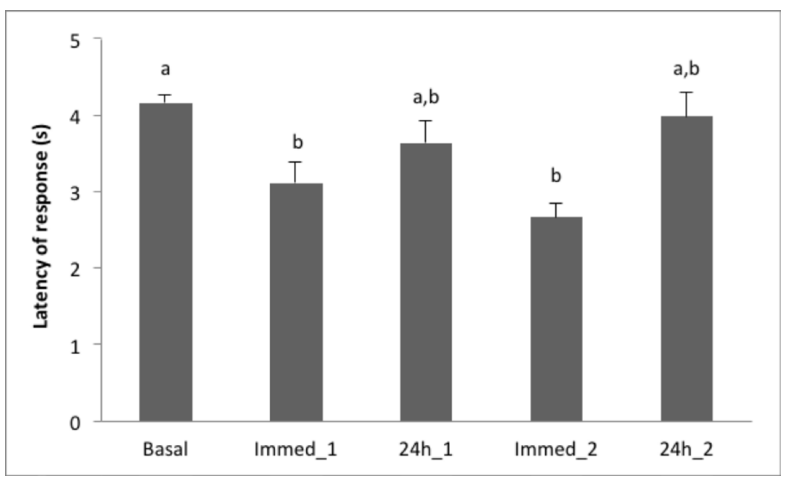

Figure 5: Evaluation of Active Sham group at different times on hot plate test. Data were expressed as the mean \pm standard error of the mean (S.E.M). Variance analysis of repeated measurements (ANOVA RM) followed by Bonferroni was performed for intra-group comparison. Different lowercase letters ( $a$ through $b$ ) indicate a statistically significant difference between the different times $(P<0.05)$. Immed_1: Immediately after first session of treatment; $24 \mathrm{~h} \_1: 24 \mathrm{~h}$ after first session of treatment; Immed_2: Immediately after last session of treatment; 24h_2: $24 \mathrm{~h}$ after last session of treatment.

\section{DISCUSSION}

We demonstrated that the procedures of immobilization of animal body and the sham stimulation (30 seconds) induced a pain threshold decrease, and our montage of the tDCS animal model was able to prevent this effect. It is important to note that the hyperalgesic effect was assessed by two behavioral components on hot plate test (paw licking and jumping), both 


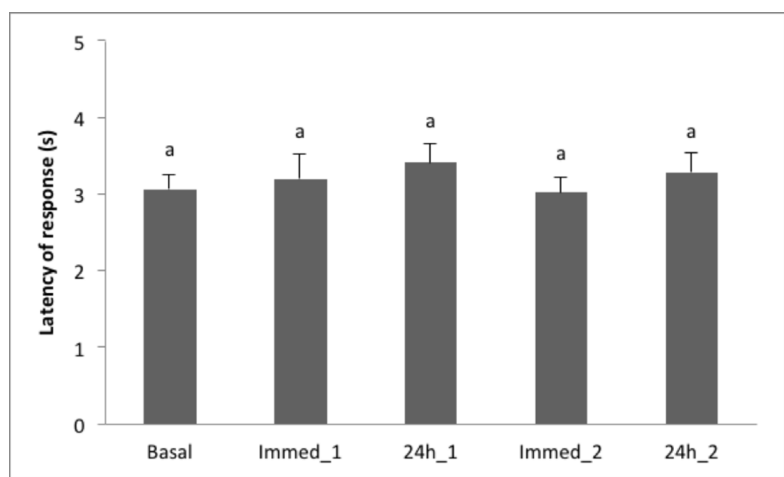

Figure 6: Evaluation of Non-active Sham group in different times on hot plate test. Data were expressed as the mean \pm standard error of the mean (S.E.M). Variance analysis of repeated measurements (ANOVA RM) followed by Bonferroni was performed for intra-group comparison. The NAS group did not present variation on different times. Equal lowercase letters (a) indicate that there is no statistically significant difference between the different times ( $P>0.05)$. Immed_1: Immediately after first session of treatment; $24 \mathrm{~h} \_1: 24 \mathrm{~h}$ after first session of treatment; Immed_2: Immediately after last session of treatment; 24h_2: $24 \mathrm{~h}$ after last session of treatment.

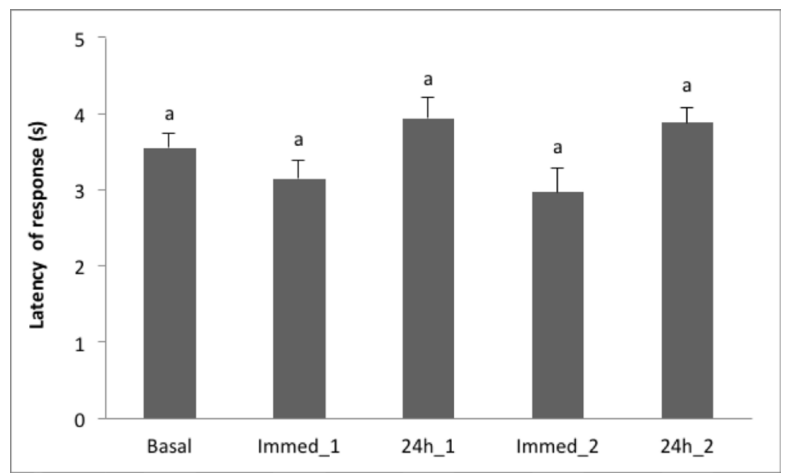

Figure 7: Evaluation of tDCS group at different times on hot plate test. Data were expressed as the mean \pm standard error of the mean (S.E.M). Variance analysis of repeated measurements (ANOVA RM) followed by Bonferroni was performed for intra-group comparison. The tDCS group did not present variation at different times. Equal lowercase letters (a) indicate that there is no statistically significant difference between the different times $(P>0.05)$. Immed_1: Immediately after first session of treatment; $24 \mathrm{~h} \_1: 24 \mathrm{~h}$ after first session of treatment; Immed_2: Immediately after last session of treatment; $24 \mathrm{~h} \_2: 2 \overline{4 h}$ after last session of treatment.

considered supraspinally-integrated responses. According to wide evidence, this constitutes the rationale for testing of the antihyperalgesic effect of tDCS. Based on the montage of our electrode, it is plausible that the sham effects (30-second stimulation) found here may be due to motor cortex stimulation. It is likely that the impact of transcranial stimulation on pain relief depends on the projection of fibers from the motor cortex to other structures involved in pain processing, such as the thalamus and brainstem nuclei that downregulate processing from sensitized neurons ${ }^{38,39}$. Besides, it is recognized that the neuromechanisms of tDCS treatment seem to induce changes in different systems.

Similarly, tDCS causes physiological changes that result in local and distant plastic changes ${ }^{40}$. Another important finding of this study is that the immobilization used in the NASR group during the tDCS treatment (20 minutes for 8 consecutive days) induced hyperalgesia ${ }^{22,41}$ indexed by the pain threshold decrease in the hot plate test. An important point is that the animals did not present analgesia immediately after 20 minutes of restraint, showing that these animals were not able to respond with stress-induced analgesia (SIA) or 20 min of restraint is not enough to promote SIA. Unlike acute stress, which has been related to a reduction in pain sensitivity, probably mediated by brainstem pain modulation ${ }^{42}$, chronic stress exposure has been associated with decreased pain threshold. Indeed, chronic stress is associated with increased sensitivity to pain, producing hyperalgesia ${ }^{19,22,43,44}$, and allodynia ${ }^{19,43}$. It is known that stress situations can activate the endogenous opioid system and promote behavioral changes, such as diminished anxiety levels, a protective mechanism of body resistance to stress ${ }^{45}$.

Furthermore, a human study has shown that a reduction in pain threshold after long-term psycho-emotional stress probably occurs due to a decrease in the activity of the brain's opioid system ${ }^{46}$. Previous data from our group suggest the involvement of the opioid system in the hyperalgesic response induced by repeated restraint stress ${ }^{22,41,44,47}$. Under these conditions, we can also suggest that data found in our study may be related to an effect of stress on the opioid system. Another possibility is that the activation of stress-related circuitry in the hypothalamus activates pain-facilitating neurons in the rostral ventromedial medulla to produce hyperalgesia, suggesting possible changes in brain activity ${ }^{42}$. It has also been postulated that the opioid system, which is an important modulator of the descending pathway of pain, could be implicated in the pain suppression mechanism ${ }^{48}$. Additionally, chronic stress is able to decrease the efficiency of the descending inhibitory systems ${ }^{49}$ and consequently facilitates the nociceptive processing. Further, it is well-known that exposure to stress situations can lead to alterations in the central dopaminergic and serotonergic nervous systems that can be involved in nociceptive response ${ }^{41,50}$, and trigger HPA axis deregulation which are associated with psychiatric disorders ${ }^{51}$. 
An interesting finding of this study is that the hyperalgesia caused by restraint stress was not lasting. Restrained rats had their pain thresholds normalized after 24 hours, being close to the baseline measurement. This effect probably occurred because eight days of restrain were not enough to produce long-lasting stress-induced hyperalgesia ${ }^{41}$. Interestingly, the 30 seconds of stimulation without immobilization-induced hyperalgesia lasted for at least 24 hours, indicating that this stimulation has a synergic stressor potential, thus we can hypothesize that active-sham potentiates the mechanisms of restraint stress. Moreover, the hyperalgesia could be related to the fact that patients with stress disorder have constant complaints of increased sensitivity to pain and other disorders involving persistent pain ${ }^{52}$. Therefore, at the clinical level, the present data could indicate that chronic pain patients need to receive a multifactorial approach to have success in the treatment. It is recognized that in the first 30 seconds of stimulation patients can feel itching and discomfort ${ }^{31}$, thus we also can propose that 30 seconds of active electric current worked like a stressor. In addition, this decreased threshold can be similar to the effect produced by a brief intense inescapable shock that can cause long-term behavioral sequelae, such as avoidance for the traumatic spatial context and increased anxiety, accompanied by increases in the nociceptive response ${ }^{53}$.

In the present study, the control and NAS groups that were just handled and the electrodes applied for 30 seconds, without active electric current, did not present variation on pain threshold. In the same way, the tDCS group, which was restrained and received 20 minutes of active stimulation, did not show variation on pain threshold. We can suggest that our montage was efficient to prevent the deleterious effect of chronic restraint stress. In this context, the analgesic effect of tDCS could be mediated by modulatory effects in pain sensation in several neurotransmitter systems, including opioid, adrenergic, substance $P$, glutamate and neurokinin receptors ${ }^{54,55}$. Viewed as a whole, the tDCS may lead to a cascade of events resulting in the modulation of synaptic neural chains that include several thalamic nuclei, the limbic system, brainstem nuclei, and the spinal cord ${ }^{56}$. The increased expression of inhibitory neurotransmitters in the dorsal horn of the spinal cord, like GABA and glycine, may explain the analgesic effects of tDCS, as observed in other neuromodulatory therapies ${ }^{57,58}$. Interestingly, brain modulation by electrical stimulation can induce changes to the endogenous opioid system in humans ${ }^{59}$. Likewise, motor cortex stimulation (MCS) induced an increase in endogenous opioid activity in patients with chronic pain ${ }^{59,60}$, and anodal tDCS enhanced endogenous opioid release in healthy humans ${ }^{3}$.

Unlike tDCS montage in humans ${ }^{1}$, the tDCS animal model is not focal, and may have a widespread effect in cortical circuits, acting on several neural centers. Among them, we can mention those involved in neuromatrix, as discussed by Melzack, reaching affective-motivational (anterior cingulate and insular cortices) and sensory-discriminative (primary and secondary somatosensorial cortices) components, acting on the pain and stress processing ${ }^{61,62}$.

In summary, the immobilization of animal body and the sham-active stimulation (30 seconds) used separately, acted like stressors decreasing pain threshold (hyperalgesia). However, when these two factors were added to the tDCS treatment this effect was prevented, demonstrating that the analgesic effect of tDCS treatment is not due to restraint stress or sham stimulation in naïve animals. In addition, the tDCS treatment was able to prevent the harmful effect of the restraint or sham-tDCS (30 seconds stimulation). Thus, our model could be safe for nociception evaluation in animals.

\section{CONCLUSIONS}

This study showed that there are methodological limitations in the present tDCS-technique, since physical restraint and 30 seconds stimulation (sham-tDCS) increased pain sensitivity. Then, the interference of those factors has to be considered during the result analysis. Although active-tDCS treatment is able to prevent these harmful effects, demonstrating that our model is a safer and effective method for tDCS application. Further studies about the tDCS-technique in rats are required to evaluate its therapeutic effects in pain and other conditions. It requires a good experimental model, without bias of sham model, and which closely resembles the model used in clinical research. Consequently, it allows the conduction translational research with potential to produce quality reliable results.

\section{Acknowledgements}

This research was supported by the following Brazilian funding agencies: the National Council for Scientific and Technological Development - CNPq (Dr. I.L.S. Torres, Dr. W. Caumo), the Foundation of Support of Research of Rio Grande do Sul, FAPERGS/PRONEM (ILS Torres - Grant 11/2050), the Graduate Research Group (GPPG) of Hospital de Clínicas de Porto Alegre - HCPA (I.L.S. Torres - Grant 100381), the Committee for the Development of Higher Education Personnel CAPES (Adachi, LNS, Laste, G), CAPES/PNPD Edital PPGCM 07/2016 (Dr. C de Oliveira); CAPES/PNPD 
Edital PPGCR 03/2016 (Dr. R Vercelino); and Grant DOCFIX FAPERGS/CAPES/09-2012 (I.C. Macedo). We also want to thank the Engineering Division from the HCPA for having developed the tDCS stimulator, MCT/FINEP - COENG/2013.

\section{Conflicts of interest}

The authors declare no conflicts of interest. The authors alone are responsible for the content and writing of the paper.

\section{REFERENCES}

1. Brunoni AR, Boggio PS, Ferrucci R, Priori A, Fregni F. Transcranial direct current stimulation: challenges, opportunities, and impact on psychiatry and neurorehabilitation. Front Psychiatry. 2013;4:19. http:// dx.doi.org/10.3389/fpsyt.2013.00019.

2. Brunoni AR, Zanao TA, Vanderhasselt MA, Valiengo L, Oliveira JF, Boggio PS, et al. Enhancement of affective processing induced by bifrontal transcranial direct current stimulation in patients with major depression. Neuromodulation. 2014;17(2):138-42. http://dx.doi.org/10.1111/ner.12080.

3. Santos MF, Love TM, Martikainen IK, Nascimento TD, Fregni F, Cummiford $C$, et al. Immediate effects of tDCS on the $\mu$-opioid system of a chronic pain patient. Front Psychiatry. 2012;3:93.

4. Hunter MA, Coffman BA, Trumbo MC Clark VP. Tracking the neuroplastic changes associated with transcranial direct current stimulation: a push for multimodal imaging. Front Hum Neurosci. 2013;7:495.

5. Lang N, Siebner HR, Ward NS, Lee L, Nitsche MA, Paulus W, et al. How does transcranial DC stimulation of the primary motor cortex alter regional neuronal activity in the human brain? Eur J Neurosci. 2005;22(2):495-504. http://dx.doi.org/10.1111/j.14609568.2005.04233.x.

6. Kuo MF, Grosch J, Fregni F, Paulus $W$, Nitsche MA. Focusing effect of acetylcholine on neuroplasticity in the human motor cortex. $J$ Neurosci. 2007;27(52):144427. http://dx.doi.org/10.1523/ JNEUROSCI.4104-07.2007.

7. Utz KS, Dimova V, Oppenländer $\mathrm{K}$, Kerkhoff G. Electrified minds: transcranial direct current stimulation (tDCS) and galvanic vestibular stimulation (GVS) as methods of non-invasive brain stimulation in neuropsychology-a review of current data and future implications Neuropsychologia. 2010;48(10):2789810. http://dx.doi.org/10.1016/j. neuropsychologia.2010.06.002.
8. Hummel FC, Cohen LG. Non-invasive brain stimulation: a new strategy to improve neurorehabilitation after stroke? Lancet Neurol. 2006;5(8):70812. http://dx.doi.org/10.1016/S14744422(06)70525-7.

9. Fregni F, Gimenes R, Valle AC, Ferreira MJ, Rocha RR, Natalle $\mathrm{L}$, et al. A randomized, shamcontrolled, proof of principle study of transcranial direct current stimulation for the treatment of pain in fibromyalgia. Arthritis Rheum. 2006c;54(12):3988-98. http://dx.doi. org/10.1002/art.22195.

10. Fregni F, Boggio PS, Nitsche MA, Marcolin MA, Rigonatti SP, Pascual-Leone A. Treatment of major depression with transcranial direct current stimulation. Bipolar Disord. 2006;8(2):203-4. http:// dx.doi.org/10.1111/j.13995618.2006.00291.x.

11. Boggio PS, Rigonatti SP, Ribeiro RB, Myczkowski ML, Nitsche MA, PascualLeone $A$, et al. A randomized, doubleblind clinical trial on the efficacy of cortical direct current stimulation for the treatment of major depression. Int J Neuropsychopharmacol. 2008;11(02):249-54. http://dx.doi. org/10.1017/S1461145707007833.

12. Brunoni AR, Baeken C, MachadoVieira R, Gattaz WF, Vanderhasselt MA. BDNF blood levels after noninvasive brain stimulation interventions in major depressive disorder: a systematic review and metaanalysis. World J Biol Psychiatry. 2015;16(2):114-22. http://dx.doi.org/10 .3109/15622975.2014.958101.

13. Fregni $F$, Boggio PS, Nitsche MA, Rigonatti SP, Pascual-Leone A. Cognitive effects of repeated sessions of transcranial direct current stimulation in patients with depression. Depress Anxiety. 2006;23(8):482-4. http://dx.doi.org/10.1002/da.20201.

14. Bae SH, Kim GD, Kim KY. Analgesic effect of transcranial direct current stimulation on central post-stroke pain. Tohoku J Exp Med. 2014;234(3):189-
95. http://dx.doi.org/10.1620/ tjem.234.189.

15. Liebetanz D, Koch R, Mayenfels $S$, König F, Paulus W, Nitsche MA. Safety limits of cathodal transcranial direct current stimulation in rats. Clin Neurophysiol. 2009;120(6):11617. http://dx.doi.org/10.1016/j. clinph.2009.01.022.

16. Nitsche MA, Paulus W. Excitability changes induced in the human motor cortex by weak transcranial direct current stimulation. J Physiol. 2000;527(3):633-9. http://dx.doi. org/10.1111/j.1469-7793.2000.t01-100633.x.

17. Bikson M, Name A, Rahman A Origins of specificity during tDCS: anatomical, activity-selective, and input-bias mechanisms. Front Hum Neurosci. 2013;7:688. http://dx.doi. org/10.3389/fnhum.2013.00688.

18. Laste G, Caumo W, Adachi LN, Rozisky JR, de Macedo IC, Filho $\mathrm{PR}$, et al. After-effects of consecutive sessions of transcranial direct current stimulation (tDCS) in rat model of chronic inflammation. Exp Brain Res. 2012;221(1):75-83. http://dx.doi. org/10.1007/s00221-012-3149-x.

19. Spezia Adachi LN, Caumo W, Laste G, Fernandes Medeiros L, Ripoll Rozisky J, Souza A, et al. Reversal of chronic stress-induced pain by transcranial direct current stimulation (tDCS) in an animal model. Brain Res. 2012;1489:17-26. http://dx.doi. org/10.1016/j.brainres.2012.10.009.

20. Spezia Adachi LN, Quevedo AS, Souza A, Scarabelot VL, Rozisky JR, Oliveira $\mathrm{C}$, et al. Exogenously induced brain activation regulates neuronal activity by top-down modulation: conceptualized model for electrical brain stimulation. Exp Brain Res. 2015;233(5):1377-89. http://dx.doi. org/10.1007/s00221-015-4212-1.

21. Kvetňansk R, Goldstein DS, Weise VK, Holmes C, Szemeredi K, Bagdy G, et al. Effects of handling or immobilization on plasma levels 
of 3,4-dihydroxyphenylalanine, catecholamines, and metabolites in rats. $J$ Neurochem. 1992;58(6):2296-302. http://dx.doi. org/10.1111/j.1471-4159.1992. tb10977.x.

22. Torres IL, Vasconcellos AP, Silveira Cucco SN, Dalmaz C. Effect of repeated stress on novelty-induced antinociception in rats. Braz J Med Biol Res. 2001;34:241-4.

23. Bali A, Jaggi AS. Preclinical experimental stress studies: protocols, assessment and comparison. Eur J Pharmacol. 2015;746:28292. http://dx.doi.org/10.1016/j. ejphar.2014.10.017.

24. Drummond GB, Paterson DJ, McGrath JC. ARRIVE: new guidelines for reporting animal research. J Physiol. 2010;588(14):2517.

25. Takano Y, Yokawa T, Masuda A, Niimi J, Tanaka S, Hironaka N. A rat model for measuring the effectiveness of transcranial direct current stimulation using fMRI. Neurosci Lett. 2011;491(1):40-3. http://dx.doi. org/10.1016/j.neulet.2011.01.004.

26. Mendonca ME, Santana MB, Baptista AF, Datta A, Bikson M, Fregni $F$, et al. Transcranial DC stimulation in fibromyalgia: optimized cortical target supported by high-resolution computational models. J Pain. 2011;12(5):610-7. http://dx.doi. org/10.1016/j.jpain.2010.12.015.

27. Nitsche MA, Cohen LG, Wassermann EM, Priori A, Lang N, Antal A, et al. Transcranial direct current stimulation: State of the art 2008. Brain Stimulat. 2008;1(3):206-23. http://dx.doi. org/10.1016/j.brs.2008.06.004.

28. Ouyang W, Hemmings HC JR. Depression by isoflurane of the action potential and underlying voltage-gated ion currents in isolated rat neurohypophysial nerve terminals. J Pharmacol Exp Ther. 2004;312(2):801-8. http://dx.doi. org/10.1124/jpet.104.074609.

29. Lu LX, Yon JH, Carter LB, JevtovicTodorovic V. General anesthesia activates BDNF-dependent neuroapoptosis in the developing rat brain. Apoptosis. 2006;11(9):1603-15. http://dx.doi.org/10.1007/s10495-0068762-3.

30. Wachter D, Wrede A, SchulzSchaeffer W, Taghizadeh-Waghefi A, Nitsche MA, Kutschenko A, et al.
Transcranial direct current stimulation induces polarity-specific changes of cortical blood perfusion in the rat. Exp Neurol. 2011;227(2):3227. http://dx.doi.org/10.1016/j. expneurol.2010.12.005.

31. Gandiga PC, Hummel FC, Cohen LG. Transcranial DC stimulation (tDCS): a tool for double-blind sham-controlled clinical studies in brain stimulation. Clin Neurophysiol. 2006;117(4):84550. http://dx.doi.org/10.1016/j. clinph.2005.12.003.

32. Woolfe G, MacDonald AD. The evaluation of the analgesic action of pethidine hydrochloride (Demerol). $J$ Pharmacol Exp Ther. 1944;80:300-7.

33. Netto CA, Siegfried B, Izquierdo I. Analgesia induced by exposure to a novel environment in rats: effect of concurrent and post-training stressful stimulation. Behav Neural Biol. 1987;48(2):304-9. http://dx.doi. org/10.1016/S0163-1047(87)90850-8.

34. Minami T, Uda R, Horiguchi S, Ito S, Hyodo M, Hayaishi O. Allodynia evoked by intrathecal administration of prostaglandin E2 to conscious mice. Pain. 1994;57(2):217-23. http://dx.doi. org/10.1016/0304-3959(94)90226-7.

35. Ossipov MH, Kovelowski CJ, Nichols ML, Hruby VJ, Porreca F. Characterization of supraspinal antinociceptive actions of opioid delta agonists in the rat. Pain. 1995;62(3):287-93. http://dx.doi. org/10.1016/0304-3959(94)00231-3.

36. Caggiula AR, Perkins KA, Saylor $S$, Epstein LH. Different methods of assessing nicotine-induced antinociception may engage different neural mechanisms. Psychopharmacology (Berl). 1995;122(3):301-6. http://dx.doi. org/10.1007/BF02246552.

37. Rubinstein M, Mogil JS, Japón M, Chan EC, Allen RG, Low MJ. Absence of opioid stress-induced analgesia in mice lacking beta-endorphin by site-directed mutagenesis. Proc Natl Acad Sci USA. 1996;93(9):39954000. http://dx.doi.org/10.1073/ pnas.93.9.3995.

38. Drouot X, Nguyen JP, Peschanski M, Lefaucheur JP. The antalgic efficacy of chronic motor cortex stimulation is related to sensory changes in the painful zone. Brain. 2002;125(7):16604. http://dx.doi.org/10.1093/brain/ awf161.
39. Lefaucheur JP. The use of repetitive transcranial magnetic stimulation (rTMS) in chronic neuropathic pain. Neurophysiol Clin. 2006;36(3):11724. http://dx.doi.org/10.1016/j. neucli.2006.08.002.

40. Medeiros LF, de Souza IC, Vidor LP, de Souza A, Deitos A, Volz MS, et al. Neurobiological effects of transcranial direct current stimulation: a review. Front Psychiatry. 2012;3:110. http:// dx.doi.org/10.3389/fpsyt.2012.00110.

41. da Silva Torres IL, Cucco SN, Bassani M, Duarte MS, Silveira PP, Vasconcellos AP, et al. Long-lasting delayed hyperalgesia after chronic restraint stress in rats: effect of morphine administration. Neurosci Res. 2003;45(3):277-83. http://dx.doi. org/10.1016/S0168-0102(02)00232-8.

42. Martenson ME, Cetas JS, Heinricher MM. A possible neural basis for stress-induced hyperalgesia. Pain. 2009;142(3):236-44. http://dx.doi. org/10.1016/j.pain.2009.01.011.

43. Bardin L, Malfetes N, NewmanTancredi A, Depoortère R. Chronic restraint stress induces mechanical and cold allodynia, and enhances inflammatory pain in rat: Relevance to human stress-associated painful pathologies. Behav Brain Res. 2009;205(2):360-6. http://dx.doi. org/10.1016/j.bbr.2009.07.005.

44. Gamaro GD, Prediger ME, Lopes J, Bassani MG, Dalmaz C. Fluoxetine alters feeding behavior and leptin levels in chronically-stressed rats. Pharmacol Biochem Behav. 2008;90(3):312-7. http://dx.doi. org/10.1016/j.pbb.2008.03.005.

45. Colasanti A, Rabiner EA, LingfordHughes A, Nutt DJ. Opioids and anxiety. J Psychopharmacol. 2011;25(11):1415-33. http://dx.doi. org/10.1177/0269881110367726.

46. Ashkinazi IY, Vershinina EA. Pain sensitivity in chronic psychoemotional stress in humans. Neurosci Behav Physiol. 1999;29(3):333-7. http:// dx.doi.org/10.1007/BF02465346.

47. Dantas G, Torres IL, Crema LM, Lara DR, Dalmaz C. Repeated restraint stress reduces opioid receptor binding in different rat CNS structures. Neurochem Res. 2005;30(1):1-7. http://dx.doi.org/10.1007/s11064-0049679-2.

48. Fields H. State-dependent opioid control of pain. Nat Rev Neurosci. 
2004;5(7):565-75. http://dx.doi. org/10.1038/nrn1431.

49. Sauro KM, Becker WJ. The stress and migraine interaction. Headache. 2009;49(9):1378-86. http://dx.doi.org/10.1111/j.15264610.2009.01486.x.

50. Gamaro GD, Manoli LP, Torres IL, Silveira R, Dalmaz C. Effects of chronic variate stress on feeding behavior and on monoamine levels in different rat brain structures. Neurochem Int. 2003;42(2):107-14. http://dx.doi.org/10.1016/S01970186(02)00080-3.

51. Nikolaus S, Antke C, Müller HW. In vivo imaging of synaptic function in the central nervous system: II. Mental and affective disorders. Behav Brain Res. 2009;204(1):32-66. http://dx.doi. org/10.1016/j.bbr.2009.06.009.

52. Asmundson GJ, Stein MB, McCreary DR. Posttraumatic stress disorder symptoms influence health status of deployed peacekeepers and nondeployed military personnel. $J$ Nerv Ment Dis. 2002;190(12):807-15. http://dx.doi.org/10.1097/00005053200212000-00002.

53. Corral-Frias NS, Lahood RP, Edelman-Vogelsang KE, French
ED, Fellous JM. Involvement of the ventral tegmental area in a rodent model of post-traumatic stress disorder. Neuropsychopharmacology. 2013;38(2):350-63. http://dx.doi. org/10.1038/npp.2012.189.

54. Morgan PJ, Barrett P, Howell HE, Helliwell R. Melatonin receptors: localization, molecular pharmacology and physiological significance. Neurochem Int. 1994;24(2):101-46. http://dx.doi.org/10.1016/01970186(94)90100-7.

55. Wu FS, Yang YC, Tsai JJ. Noncompetitive inhibition of the glycine receptor-mediated current by melatonin in cultured neurons. Brain Res. 2000;881(2):208-11. http://dx.doi. org/10.1016/S0006-8993(00)02804-3.

56. Lima MC, Fregni F. Motor cortex stimulation for chronic pain: systematic review and meta-analysis of the literature. Neurology. 2008;70(24):232937. http://dx.doi.org/10.1212/01. wnl.0000314649.38527.93.

57. Han JS. Acupuncture: neuropeptide release produced by electrical stimulation of different frequencies. Trends Neurosci. 2003;26(1):17-22. http://dx.doi.org/10.1016/S01662236(02)00006-1.
58. Wen G, He X, Lu Y, Xia Y. Effect of acupuncture on neurotransmitters/ modulators. In: Ying X, Xioding C, Gencheng W, Jieshu C, editors. Acupuncture therapy for neurological diseases: a neurobiological view. London: Springer Heidelberg Dordrecht; 2010. p. 120-42.

59. Taylor JJ, Borckardt JJ, George MS. Endogenous opioidsmediate left dorsolateral prefrontal cortex rTMS-induced analgesia. Pain. 2012;153(6):1219-25. http://dx.doi. org/10.1016/j.pain.2012.02.030.

60. Maarrawi J, Peyron R, Mertens P, Costes N, Magnin M, Sindou $\mathrm{M}$, et al. Motor cortex stimulation for pain control induces changes in the endogenous opioid system. Neurology. 2007;69(9):827-34. http://dx.doi.org/10.1212/01. wnl.0000269783.86997.37.

61. Melzack R. Phantom limbs and the concept of a neuromatrix. Trends Neurosci. 1990;13(3):88-92. http://dx.doi.org/10.1016/01662236(90)90179-E.

62. Moseley GL. A pain neuromatrix approach to patients with chronic pain. Man Ther. 2003;8(3):130-40. http://dx.doi.org/10.1016/S1356689X(03)00051-1.

Received: Feb 20, 2017 Accepted: Apr 04, 2017 